\title{
Post-irradiation Brain Tumors
}

\author{
Shinichiro OKAMOTO, Hajime HANDA, Junkoh YAMASHITA, \\ Yasuhiko TOKURIKI and Mitsuyuki ABE*
}

\author{
Departments of Neurosurgery and ${ }^{*}$ Radiology, \\ Kyoto University Medical School, Kyoto
}

\begin{abstract}
Four cases of brain tumor - three meningiomas and one glioblastoma - that developed after radiation therapy are reported. The location and course of each tumor were highly suggestive of a pathogenetic role of the preceding irradiation in the development of the tumor. A review of the literature revealed that there is evidence for a causative role of irradiation in the development of brain tumors, although there has been no definitive epidemiological study.
\end{abstract}

Key words: brain neoplasms, post-irradiation neoplasms, meningioma, glioblastoma, radiation therapy

\section{Introduction}

It is well known that ionizing radiation has oncogenetic activity. In the central nervous system, the tumors that most commonly develop after therapeutic irradiation, and are thus suspected of being radiation-induced, are fibrosarcomas. ${ }^{4,20}$ Reports of meningiomas or gliomas following irradiation are rare. In this report, we will present four cases of brain tumors - three meningiomas and one glioblastoma - that strongly suggest an oncogenetic role for irradiation.

\section{Case Reports}

\section{〈Case 1)}

The details of this case have been reported previously. ${ }^{25)}$ The patient was admitted to our hospital for the first time on April 8, 1963, at 23 years of age, because of headache and amenorrhea. A suprasellar cystic tumor was partially removed through a transcranial, transventricular route, with good results. The histological diagnosis was craniopharyngioma. Because the surgery was non-radical, the remaining tumor was subsequently irradiated with ${ }^{60} \mathrm{Co}$. A cumulative dose of $56 \mathrm{~Gy}$ was given to the head by a rotating method over 40 days, in 31 fractions. The patient did well for 13 years. At age 36 she began to complain of headache, memory dis-

Received October 2, 1984; Accepted January 7, 1985 turbance, and urinary incontinence. She was readmitted to our hospital on January 10, 1975, and a right tentorial meningioma was removed. The histological diagnosis was nonmalignant fibroblastic meningioma.

\section{$\langle$ Case 2〉}

This 44-year-old female had previously been admitted to our hospital on November 4, 1975, at 39 years of age, because of decreased visual acuity and temporal hemianopsia of the left eye. She had also been amenorrheic for 1 year prior to admission. Neurological examination disclosed bitemporal hemianopsia and papilledema of the right optic fundus. Plain skull films and pneumoencephalograms revealed a mass lesion in and above the sella. A solid tumor in the sella extending upward and compressing the optic nerves was partially removed through a right subfrontal approach. The histological diagnosis was chromophobe pituitary adenoma. The patient then received ${ }^{60} \mathrm{Co}$ irradiation in a total dose of $50 \mathrm{~Gy}$ to the sellar region, of which $30 \mathrm{~Gy}$ was administered through opposing $5 \times 5 \mathrm{~cm}$ lateral ports and the remaining $20 \mathrm{~Gy}$ through rotating ports. The dose was delivered over 54 days in 28 fractions. She was discharged in good condition and did well for 5 years. A follow-up computerized tomographic (CT) scan taken 9 months before readmission did not show any abnormality.

Five years after her first admission, she began to complain of general malaise, headache, and weakness of the right upper extremity. The neurological examination on readmission (July 23, 1980) revealed 

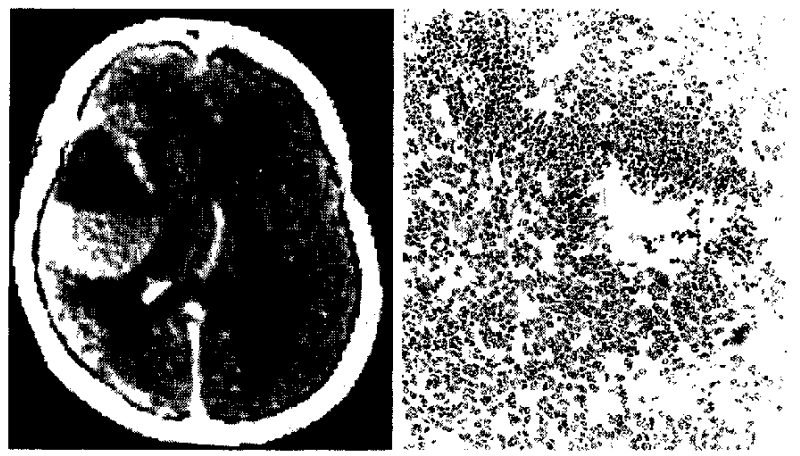

Fig. 1 Case 2. left: A CT film taken 5 years after irradiation for a pituitary adenoma, showing a large, cystic mass with ring-like enhancement in the left temporal lobe, which represents niveau by intratumoral hemorrhage. right: $\mathrm{A}$ photomicrograph of the surgical specimen, showing pseudopallisading around a necrotic center, endothelial proliferation, prominant mitosis, and pleomorphism, all of which are indicative of glioblastoma. HE stain, $\times 100$.

bilateral moderately choked discs, paresis and hypesthesia of the right side, and logorrhea. A CT scan revealed a large, cystic mass in the left temporal lobe, with severe perifocal edema. The tumor was enhanced by contrast medium in a ring-like fashion and represented niveau by intratumoral hemorrhage (Fig. 1 left $)$. A left carotid angiogram revealed heterogeneous tumor staining in the arterial phase.

A left frontoparietotemporal craniotomy was performed. Approximately $50 \mathrm{ml}$ of bloody fluid was evacuated and the solid portion of the tumor was macroscopically totally removed, although it was poorly demarcated from the surrounding brain tissue. The histological diagnosis was glioblastoma (Fig. 1 right).

The patient was discharged in good condition. However, she again began to complain of headache, and recurrence of the tumor was evident on a $\mathrm{CT}$ scan taken 2 months after discharge. Conservative therapy was administered at another hospital but she died 14 months after the second operation.

\section{$\langle$ Case 3〉}

This 36-year-old female had received radium therapy for a hemangioma of the right forehead scalp when she was an infant. Although the precise radiation dose was unknown, her mother clearly remembered the course of events. After the completion of radiation therapy, a small scar remained on her scalp. She was well until 3 weeks before admission, when she began to complain of headache and visual disturbance. Neurological examination on ad-
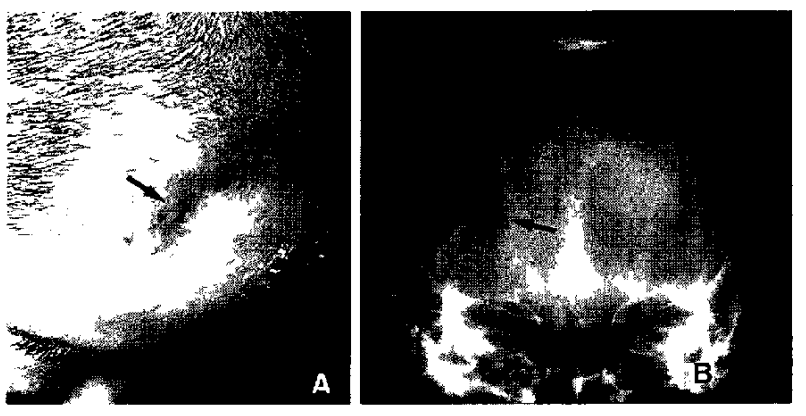

Fig. 2 Case 3. A: A scar in the right forehead (arrow) which developed after radiation therapy for a hemangioma in infancy. B: A plain skull film obtained on admission, showing an osteolytic lesion just beneath the scar (large arrow). An increased vascular groove due to a feeding artery (small arrows) is also evident.

mission (June 27, 1983) revealed bilateral choked discs, decreased visual acuity of the right eye, and recent memory disturbance. A scar was noted on the right forehead scalp (Fig. 2A). Plain skull films showed an oval-shaped osteolytic lesion surrounded by an osteoplastic area in the right frontal calvarium, just beneat the scar. An enlarged vascular groove due to a dilated branch of the middle meningeal artery, was also evident (Fig. 2B). A CT scan revealed a huge mass of slightly high density occupying nearly half of the right anterior fossa and severe perifocal edema. The tumor was markedly and homogeneously enhanced by contrast medium (Fig. 3 left). A right carotid angiogram disclosed the characteristic sunburst appearance in the affected region.

A right frontotemporal craniotomy was performed, and a solid extra-axial tumor was totally removed. The tumor was attached to the dura of the right frontal convexity, where it had eroded the overlying skull and reached to the subperiosteal region. The tumor attachment was just beneath the scar of the skin. The histological diagnosis was fibroblastic meningioma (Fig. 3 right).

The postoperative course has been uneventful, and there has been no recurrence for 9 months.

\section{Case 4}

This 28-year-old male was first admitted to our hospital on June 24,1967 , at 12 years of age, because of headaches and tremor in the right hand, both of 2 years' duration. Neurological examination disclosed a fine tremor of the right hand and horizontal nystagmus. There was an abnormal calcification in the diencephalic region on plain skull films. Both pneumoencephalograms and the left carotid angiograms were suggestive of a deep frontal mass just lat- 

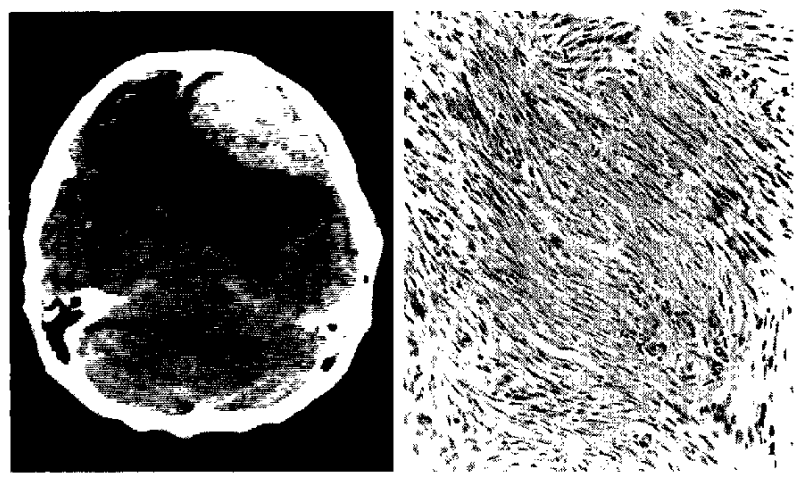

Fig. 3 Case 3. left: A CT film with contrast enhancement on admission, 36 years after radiation therapy. Note the large, well circumscribed mass with homogeneous enhancement in the right frontal region. right: A photomicrograph of the surgical specimen. The histological diagnosis was fibroblastic meningioma without any malignant figure. HE stain, $\times 200$.
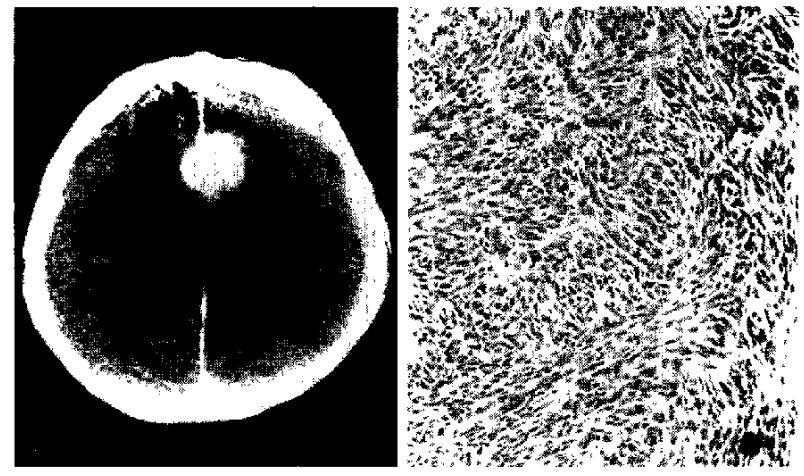

Fig. 4 Case 4. left: A CT film with contrast enhancement taken 16 years after irradiation for an astrocytoma in the left basal ganglia. Note a round, homogeneously enhanced mass attached to the falx. right: A photomicrograph of the surgical specimen. The histological diagnosis was nonmalignant transitional meningioma. HE stain, $\times 200$.

eral to the anterior horn of the left lateral ventricle. A solid tumor embedded under the ependyma and reaching into the left caudate head was partially removed. The histological findings were consistent with those of astrocytoma. Subsequently he received radiation therapy with ${ }^{60} \mathrm{Co}$ to the left frontal lobe. A total dose of $55 \mathrm{~Gy}$ was delivered over 41 days in 30 fractions.

He was healthy until 6 years later, when he experienced a generalized seizure. Similar attacks occur- red several times in the following 10 years, despite anticonvulsant medication. Although follow-up CT scans obtained 9 and 12 years after surgery showed no abnormality, those taken 16 years after the operation disclosed regrowth of the previous tumor as well as a new mass lesion. He was readmitted to our hospital on February 14, 1983.

The neurological examination was negative except for the persistence of the hand tremor. CT films showed a partially calcified, isodense mass in the subependymal region of the left caudate head and the anterosuperior aspect of the left thalamus, which was not enhanced by contrast medium. In addition, on the midline just above the former mass, there was another spherical, isodense mass attached to the falx, and this mass was markedly and homogeneously enhanced by contrast medium (Fig. 4 left).

A bifrontal craniotomy was performed and a well circumscribed, solid tumor was totally removed, along with its falcial attachment. At the same time, a recurrent astrocytoma was partially removed. The histological diagnosis of the new tumor was transitional, nonmalignant meningioma (Fig. 4 right).

He was discharged in good condition and there has been no recurrence for 13 months.

\section{Discussion}

A tumor that is postulated to be radiation-induced must fulfill all of the following criteria: ${ }^{3,24}$ a) the tumor must have developed in the irradiated area; b) it must not have been present prior to irradiation; c) there must be a latent period; and d) the tumor must be verified histologically. Our four cases apparently fulfill these criteria.

Recently, Iwai et al. ${ }^{14)}$ collected 73 cases of radiation-induced meningiomas. Radiation-related gliomas are far rarer; only 17 cases have been reported in the literature. ${ }^{22)}$ It is unclear why tumors that develop after irradiation to the central nervous system tend to be mesodermal in origin, as are fibrosarcomas and meningiomas, rather than glial in origin.

of the reported cases that fulfill the above mentioned criteria for radiation-induced tumors, six reported meningiomas ${ }^{2,10,14,24,27}$ (including our Case 3) that developed after radiation therapy of hemangiomas or vascular nevi of the scalp are particularly interesting because of the close relationships between the sites of irradiation and the dural attachments of the tumors (Table 1). An extraordinary feature of our Case 3 is that the dural attachment of the meningioma was just beneath the scar that resulted from irradiation with radium. It is improbable that this occurred merely by chance. None of 
Table 1 Meningiomas following radiation therapy for scalp vascular lesions

\begin{tabular}{|c|c|c|c|c|}
\hline Author (Year) & Age/Sex & Site of primary lesion & $\begin{array}{l}\text { Radiation } \\
\text { source }\end{array}$ & Site of meningioma \\
\hline Feiring \& Foer $(1968)^{10)}$ & $30 / \mathrm{F}$ & It. frontoparietal region & radium & lt. suprasylvian region \\
\hline $\begin{array}{l}\text { Bogdanowicz \& Sachs } \\
(1974)^{2)}\end{array}$ & $59 / \mathrm{M}$ & lt. face to It. temple & radium & It. sphenoid ridge \\
\hline Watts $(1976)^{27)}$ & $23 / F$ & rt. face and head & X-ray & rt. sphenoid ridge \\
\hline $\begin{array}{l}\text { Spallone et al. } \\
(1979)^{24)}\end{array}$ & $25 / M$ & rt. parieto-occipital region & $X$-ray & $\begin{array}{l}\text { rt. parieto-occipital } \\
\text { convexity }\end{array}$ \\
\hline Iwai et al. $(1984)^{14)}$ & $49 / \mathrm{M}$ & rt. frontoparietal region & $?$ & rt. frontal convexity \\
\hline Present case (Case 3) & $36 / \mathrm{F}$ & rt. forehead & radium & rt. frontal convexity \\
\hline
\end{tabular}

Table 2 Glioblastomas following radiation therapy

\begin{tabular}{|c|c|c|c|c|}
\hline Author (Year) & Age/Sex & $\begin{array}{l}\text { Dose-Time- } \\
\text { Fractionation* }\end{array}$ & NSD (rets) & $\begin{array}{l}\text { Latency } \\
\text { (years) }\end{array}$ \\
\hline Komaki et al. (1977) ${ }^{17)}$ & $28 / \mathrm{M}$ & $5,400-(37)-(27)$ & 1,628 & 6 \\
\hline Clifton et al. $(1980)^{6}$ & $21 / \mathrm{M}$ & $4,969-22-16$ & 1,799 & 6 \\
\hline Pearl et al. $(1980)^{211}$ & $5 / \mathrm{M}$ & $3,000-(21)-(15)$ & 1,123 & 13 \\
\hline Chung et al. $(1981)^{5)}$ & $2 / \mathrm{M}$ & $2,400-15-12$ & 977 & 5 \\
\hline Barnes et al. $(1982)^{1)}$ & $17 / \mathrm{F}$ & $4,000-(28)-20$ & 1,352 & 6 \\
\hline Piatt et al. $(1983)^{22)}$ & $38 / \mathrm{M}$ & $4,900-(38)-25$ & 1,510 & 14 \\
\hline Present case (Case 2) & $44 / \mathrm{F}$ & $5,000-54-28$ & 1,440 & 5 \\
\hline Mean \pm SEM & & 4,238 & $1,404 \pm 107.5$ & 7.9 \\
\hline
\end{tabular}

*Dose and time are represented in cGy and days, respectively. It was assumed that the conventional method (200 cGy in each fraction, 5 days a week) was applied if the information in the literature was insufficient. The presumptive data are shown in parentheses. NSD: nominal standard dose.

these cases exhibited any feature of phacomatosis, in which meningiomas and cutaneous vascular lesions may coexist.

The latency between radiation therapy and tumor diagnosis may be estimated according to the radiation dosage, degree of malignancy of the tumor, and location of the tumor. It has been suggested that larger radiation dosages and higher degrees of malignancy are associated with a shorter latency. The mean latencies reported are 20.8 years for meningiomas with high-dose radiation (over $10 \mathrm{~Gy}$ ) and 31.3 years for those with low-dose radiation (under $10 \mathrm{~Gy}$ ), ${ }^{13)}$ 11.1 years for gliomas, ${ }^{22)}$ and 10 years for fibrosarcomas. ${ }^{26)}$ The mean radiation dosage and mean latency in seven cases of glioblastomas ${ }^{1,5,6,17,21,22)}$ (including our Case 2) were calculated to be $4,238 \mathrm{cGy}$ $(2,400$ to $5,400 \mathrm{cGy})$ and 7.9 years (5 to 14 years) (Table 2 ). In addition, the diagnosis may be delayed if the tumor develops in a relatively silent area, as happened in our Case 3.

It has been well established that the most important factors in the biological response to irradiation include total dose, size and number of individual treatment fractions, overall treatment time, and the volume irradiated. ${ }^{23)}$ Thus, the concept of nominal standard dose (NSD) has been advocated. ${ }^{8)}$ It was calculated from the formula

$$
\text { NSD (rets) }=D \times N^{-0.24} \times T^{-0.11}
$$

where $D$ is the total dose, $N$ is the number of fractions, and $T$ is the total treatment time in days. The mean NSD value for 78 patients with radiation necrosis, collected by Sheline, ${ }^{23)}$ was $2,017 \pm 42.8$ rets. The value for post-irradiation glioblastomas, summarized in Table 2, was $1,404 \pm 107.5$ rets, which was significantly lower than the NSD for radiation necrosis $(p<0.001$, Student's T test). The NSD values in our two cases of meningioma, Cases 1 and 4 , were 1,651 and 1,597 rets, respectively.

The meningiomas that developed after radiation therapy by the Kienbock-Adamson method for Tinea capitis make up the vast majority of radiationrelated meningiomas. These individuals received smaller doses (usually $800 \mathrm{R})^{24)}$ and thus had smaller NSDs than did patients irradiated for primary neoplasms of the head and neck. These facts imply that relatively low doses may be sufficient to induce secondary neoplasms in the central nervous system. They 
also suggest that different mechanisms may be involved in the two distinct late effects of irradiation, i.e., radiation necrosis and secondary neoplasms.

Our cases and others reported in the literature are suggestive of the oncogenicity of irradiation to the central nervous system. However, the hypothesis that irradiation is one of the oncogenetic factors in brain tumors will be proven only when the following issues are clarified by sophisticated investigations: 1) Do epidemiological studies confirm that irradiation is a risk factor in brain tumor patients? 2) Does statistical analysis indicate that the incidence of brain tumors increases with increasing radiation doses? 3) Is it possible to produce brain tumors under well controlled experimental conditions? For certain carcinomas or sarcomas, the role of irradiation in the development of tumors has been well established. ${ }^{9)}$ However, such studies of brain tumors have been very few. ${ }^{11,19)}$ Modan et al. ${ }^{19\}}$ concluded that the incidence of tumors of the head and neck was significantly higher in 11,000 children irradiated for scalp ringworm than in the same number of non-irradiated children in a 12 - to 24 -year follow-up period.

The relationship between radiation dose and incidence of brain tumors is still to be defined. Several reports have described the development of brain tumors similar to human glioblastoma in the irradiated brains of primates. ${ }^{12,16,18)}$ The induction of meningioma in experimental models is still controversial. $^{7,15)}$

\section{References}

1) Barnes AE, Liwnicz BH, Schellhas HF, Altshuler G, Aron BS, Lippert WA: Successful treatment of placental choriocarcinoma metastatic to brain followed by primary brain glioblastoma. Gynecol Oncol 13: 108-114, 1982

2) Bogdanowicz WM, Sachs E Jr: The possible role of radiation in oncogenesis of meningioma. Surg Neurol 2: $379-383,1974$

3) Cahan WG, Woodard HQ, Higinbotham NL, Stewart FW, Coley BL: Sarcoma arising in irradiated bone. Cancer 1: 3-29, 1948

4) Chasman LR, Robertson DC, Farmer AW: Irradiation fibrosarcoma. Plast Reconstr Surg 20: 55-61, 1957

5) Chung CK, Stryker JA, Cruse R, Vannuci R, Towfighi J: Glioblastoma multiforme following prophylactic cranial irradiation and intrathecal methotrexate in a child with acute lymphocytic leukemia. Cancer 47: 2563-2566, 1981

6) Clifton MD, Amromin GD, Perry MC, Abdir R, Watts C, Levy N: Spinal cord glioma following ir- radiation for Hodgkin's disease. Cancer 45: 20512055,1980

7) Dimant JN, Loktinov GM, Sataev MM: Induction of the spinal cord meningeal tumors in rabbits with radioactive cobalt. Vop Onkol 11: 46-51, 1965

8) Ellis F: Dose, time and fractionation. A clinical hypothesis. Clin Radiol 20: 1-7, 1969

9) Fajardo LF: Pathology of Radiation Injury. New York, Masson, 1982, pp 252-255

10) Feiring EH, Foer WH: Meningioma following radium therapy. Case report. J Neurosurg 29: 192194,1968

11) Gold E, Gordis L, Tonascia J, Szklo M: Risk factors for brain tumors in children. Am J Epidemiol 109 : 309-319, 1979

12) Hymaker W, Rubinstein LJ, Miquel J: Brain tumors in irradiated monkeys. Acta Neuropathol (Berl) 20: $267-277,1972$

13) Iacono RP, Apuzzo MLJ, Davis RL, Tsai FY: Multiple meningiomas following radiation therapy for medulloblastoma. Case report. I Neurosurg 55: 282286, 1981

14) Iwai $T$, Hattori $T$, Sakai $N$, Yamada $H$ : Meningioma following irradiation for vascular nevus. A case report. No Shinkei Geka 12: 499-504, 1984 (in Japanese)

15) Janisch W, Kirsch M: Tierversuche zur Induktion von Intrakraniellen Geschwulsten durch Ionisierende Strahlen. Exp Pathol (Jena) 1: 226-233, 1967

16) Kemper TL, O'Neill RR, Caveness WF: Effects of single dose supervoltage whole brain radiation in Macaca mulatta. J Neuropathol Exp Neurol 36: 916940,1977

17) Komaki S, Komaki R, Choi H, Correa-Paz F: Radiation- and drug-induced intracranial neoplasm with angiographic demonstration. Neurol Med Chir (Tokyo) 17 [Part I]: 55-62, 1977

18) Krupp JH: Nine-year mortality experience in protonexposed Macaca mulatta. Radiat Res 67: 244-251, 1976

19) Modan B, Baidatz D, Mart H, Steinitz R, Sevin SG: Radiation-induced head and neck tumours. Lancet 1 : 277-279, 1974

20) Norwood CW, Kelly DL Jr, Davis CH Jr, Alexander E Jr: Irradiation-induced mesodermal tumors of the central nervous system: Report of two meningiomas following X-ray treatment for gliomas. Surg Neurol 2: $161-164,1974$

21) Pearl GS, Mirra SS, Miles ML: Glioblastoma multiforme occurring 13 years after treatment of a medulloblastoma. Neurosurgery 6: 546-551, 1980

22) Piatt JH Jr, Blue JM, Schold SC Jr, Burger PC: Glioblastoma multiforme after radiotherapy for acromegaly. Neurosurgery 13: 85-89, 1983

23) Sheline GE: Irradiation injury of the human brain: A review of clinical experience, in Gilbert HA, Kagan AR (eds): Radiation Damage to the Nervous System. A Delayed Therapeutic Hazard. New York, Raven, 1980, pp 39-58 
24) Spallone A, Gagliardi FM, Vagnozzi R: Intracranial meningiomas related to external cranial irradiation. Surg Neurol 12: 153-159, 1979

25) Waga $\mathrm{S}$, Handa $\mathrm{H}$ : Radiation-induced meningioma: With review of literature. Surg Neurol 5: 215-219, 1976

26) Waltz TA, Brownell B: Sarcoma: A possible late result of effective radiation therapy for pituitary adenoma. Report of two cases. J Neurosurg 24: $901-$ 907,1966
27) Watts $C$ : Meningioma following irradiation. Cancer 38: 1939-1940, 1976

Address reprint requests to: S. Okamoto, M.D., Department of Neurosurgery, Kyoto University Medical School, 54 Kawahara-cho, Shogoin, Sakyo-ku, Kyoto 606, Japan. 